\title{
Biofilms: Microbes and Disease
}

\author{
Aparna, Madhu Sharma Pt.B.D. and Sarita Yadav \\ Department of Microbiology, Sharma PGIMS, Rohtak; Haryana, India
}

\begin{abstract}
Bacteria that attach to surface aggregate in a hydrated polymeric matrix of their own synthesis to form biofilms. These represent microbial societies with their own defense and communication system. Transitioning from acute to chronic infection is frequently associated with biofilm formation.Bacteria in biofilms are innately more resistant to antimicrobial agents. The presence of indwelling medical devices increases the risk for biofilm formation and subsequent infection. The current antibiotic therapies are of limited effectiveness in resolving biofilms infection.This review attempts to discuss the stages in biofilm formation, their pathogenic mechanisms, effect of antimicrobial agents, detection and eradication of the biofilms.
\end{abstract}

Key-Words: Biofilms, staphylococci, Pseudomonas, Candida.

The concept of bacteria living within the context of a community rather than simply as autonomous entities is one that is quickly gaining acceptance. These communities of organisms living within extracellular matrix are known as biofilms.They can develop on abiotic and biotic surfaces, acting as a source of various infections. Biofilm development on surfaces is a dynamic stepwise process involving adhesion,growth,motility and extracellular polysaccharide production. The nature of biofilm and the physiological state of bacterial cells within the biofilm confers high level of resistance to antimicrobial agents. With the emergence of biofilm associated diseases, there are considerable diagnostic problems for the clinical laboratory. So,various techniques for detection and eradication of biofilms have been described.

Perhaps because many biofilms are sufficiently thick to be visible to the naked eye, these microbial communities were among the first to be studied by the late developing science of microbiology. Anton Van Leeuwenhoek scraped the plaque biofilm from his teeth and observed the "animalculi" that produced this microbial community with his primitive microscope. However, it was not until the 1970's that we began to appreciate that bacteria in the biofilm mode of existence, sessile bacteria, constitute a major component of the bacterial biomass in many environments, and it was not until the 1980s and 1990s that we began to appreciate that attached bacteria were organized in elaborate ways [1]. For e.g., different bacterial species specifically attach to different surfaces or co-aggregate with specific partners in the mouth. Often one species can coaggregate with multiple partners, which themselves can aggregate with other partner to form a dense bacterial plaque. Advances in light microscopy coupled with developments in microelectrode technology have led to an appreciation that bacterial biofilms consist of microcolonies on a surface, and that within these microcolonies the bacteria have developed

Received on 7 August 2008; revised 28 November 2008.

Address for correspondence: Dr. Aparna. Associate Professor. Department of Microbiology. Pt. B.D. Sharma PGIMS, Rohtak 124001. Haryana - India. E-mail: aparnaparmar2008@rediffmail.com.

The Brazilian Journal of Infectious Diseases

2008;12(6):526-530. (C) 2008 by The Brazilian Journal of Infectious Diseases and Contexto Publishing. All rights reserved. into organised communities with functional heterogenicity.

The organisms in a biofilm are specialised and have great deal of genetic energy. Given the selected pressures of certain environments, notably aquatic systems, biofilms are preferred method of growth. Their unique structure that evolves over time allows for a cohesive, robust community of cells with interspecies communication driven by the principle of survival. Microorganisms, be they are prokaryotic or eukaryotic, have the potential to live in one of the two phenotypes: sessile or planktonic. The sessile phenotype result from attachment and usually develops into a multispecies biofilm that has unique characteristics, making it similar in many ways to hydrated polymers. Planktonic are free - floating microorganisms [2]. These sessile bioflm communities can give rise to non sessile individuals, planktonic bacteria that can rapidly multiply and dispose. The common view is that planktonic bacteria must expose themselves to deleterious agents in their environment, be they phage or amoeba in nature, biocides in industrial settings or potent antimicrobial agents in a clinical setting. Since biofilms contaminate industrial pipelines, dental unit water lines, catheters, ventilators and medical implants, they act as a source of disease for humans, animals and plants. In this light, it is not surprising that an impressive number of chronic bacterial infections involve bacterial biofilms, which are not easily eradicated by conventional antibiotic therapy [1].

\section{Stages in Biofilm Development}

Biofilms like other communities form gradually over time. There is a five stage universal growth cycle of a biofilm with common characteristics independent of the phenotype of the organisms. Stage 1 is the attachment phase that can take only seconds to activate and is likely induced by environmental signals. These signals vary by organisms but they include changes in nutrients and nutrient concentrations, $\mathrm{pH}$, temperature, oxygen concentration, osmolality and iron. Rough surfaces are more susceptible to biofilm formation this is likely due to reduction of shear forces and increased surface area. Studies indicate that biofilms also tend to form more readily on hydrophobic materials like teflon and other plastics than on glass and metal. The initial binding in stage I is reversible as some cells detach have the substraction. During 
this stage, bacterial cells exhibit a logarithmic growth rate.

Stage II is characterized as irreversible binding and begins minutes after stage 1 . After adhering to the epithelial surface, the bacteria begin to multiply while emitting chemical signals that "inter communicate" the bacterial cells. Once the signal intensity exceeds a certain threshold level, the genetic mechanisms underlying exopolysaccharide (EPS) production are activated which is able to trap nutrients and planktonic bacteria [1]. During stage II cell aggregates are formed and motility is decreased when cell aggregates become progressively layered with a thickness greater than $10 \mu \mathrm{m}$, the biofilm is in stage III also known as maturation I. When biofilms reach their ultimate thickness generally greater than $100 \mathrm{~mm}$, this is called stage IV or maturations - 2. During stage V, cell dispersion is noted. Some of the bacteria develop the planktonic phenotype and leave the bioflm. This begins several days after stage IV [2].

Although certain constituents are common to all bioflms, the contribution of the host relative to the microorganisms such as immunologic components and the physical locations have an impact on this structure. Several key environmental and cultural characteristics affect the selection of multispecies biofilm inhabitants (Figure 1)

Much of the development and structural integrity of the

Figure 1. Environmental and cultural characteristics which affect the selection of biofilms multispecies.

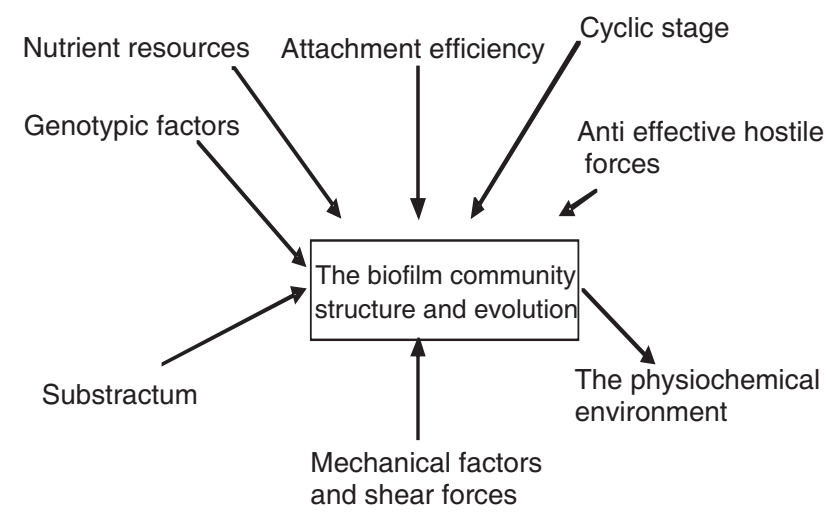

biofilm is dependent upon quorum sensing (QS). QS is primarily a means with which extracellular molecules, pheromones, enhance communication among bacteria. The viability of the biofilm community is dependent upon stress response genes and cell signaling from the cells via QS or quorum diffusions. In P. aeruginosa it appears that an acylated homoserine lactone (acyl-HSL) is an important player in this type of cell to cell signaling [3]. Quorum sensing is widespread among several pathogenic and non pathogenic genera. Emerging evidence points to the involvement of quorum sensing in biofilm formation and surface motility in the opportunistic pathogens, Pseudomonas aeruginosa, Burkhoederia cepacia and Aeromonas hydrophilia. Quorums sensing genes are critical for pathogenesis of $P$. aeruginosa infection in the cystic fibrosis lung [4].

The three dimensional architecture of the mature biofilm has three layers and is comprised of stalks of mushroom shaped microcolonies attached to the substractum surrounded by EPS. The biofilm matrix contains EPS, proteins and DNA; EPS constituents 50\% to 90\% of the organic carbon in the matrix. Many of the stalks and mushrooms together result in an architecture with water channels between the bacterial clusters. The water channels have been likened to a primitive circulatory system which protects cell bacteria against buildup of toxic metabolites and starvation while providing a source of nutrients [5].

\section{Pathogenic Mechanisms}

Different pathogenic mechanisms of the biofilms have been proposed. These include:

- Allow attachment to a solid surface;

- "Division of labor" increases metabolic efficiency of the community;

- Evade host defenses such as phagocytosis;

- Obtain a high density of microorganisms;

- Exchange genes that can result in more virulent strains of microorganisms;

- Produce a large concentration of toxins;

- Protect from antimicrobial agents;

- Detachment of microbial aggregates transmits microorganisms to other sites.

Biofilms develop preferentially on inert surfaces or on dead tissue, and occur commonly on medical devices and fragments of dead tissue such as sequestra of dead bone; they can also form on living tissues, as in the case of endocarditis [6]. Sessile bacterial cells release antigens and stimulate the production of antibodies, but the antibodies are not effective in killing bacteria within biofilms and may cause immune complex damage to surrounding tissues. Even in individuals with excellent cellular and humoral immune reactions, biofilm infections are rarely resolved by the host defense mechanisms [7].

More than half of the infectious diseases that affect mildly compromised individuals involves bacterial species that are commensals with the human body or are common in our environments. The surfaces of medical devices have been foci of device related infections showing the presence of large number of slime encased bacteria as evidenced by electron microscopy. Even the tissues taken from non device related chronic infections also show the presence of biofilm formation. These biofilm infections may be caused by a single species or by a mixture of species of bacteria or fungi (Table 1) [1,2].

\section{Biofilms and Antimicrobial Agents}

The armament of therapeutic agents available to treat bacterial infections today is restricted to antibiotics developed specifically to kill or stop the growth of individual bacteria. 
Table 1. Partial list of human infections involving biofilms.

\begin{tabular}{|c|c|}
\hline Infection or disease & Common biofilm bacterial species \\
\hline Dental caries & Acidogenic Gram-positive cocci (e.g. Streptococcus) \\
\hline Periodontitis & Gram-negative anaerobic oral bacteria \\
\hline Otitis media & Nontypable strains of Haemophilus influenzae \\
\hline Musculoskeletal infections & Gram-positive cocci (e.g., Staphylococci) \\
\hline Necrotizing fascitis & Group A Streptococci \\
\hline Biliary tract infection & Enteric bacteria (eg., Escherichia coli) \\
\hline Osteomyelitis & Various bacterial and fungal species - often mixed \\
\hline Bacterial prostatitis & E. coli and other Gram-negative bacteria \\
\hline Native valve endocarditis & Viridans Group Streptococci \\
\hline Cystic fibrosis pneumonia & P. aeruginosa and Burkholderia cepacia \\
\hline Meliodiosis & Pseudomonas pseudomallei \\
\hline \multicolumn{2}{|l|}{ Nosocomial infections } \\
\hline ICU pneumonia & Gram-negative rods \\
\hline Sutures & Staphylococcus epidermidis and S. aureus \\
\hline Exit sites & S. epidermidis and S. aureus \\
\hline Arteriovenous shunts & S. epidermidis and $S$. aureus \\
\hline Schleral buckles & Gram-positive cocci \\
\hline Contact lens & P. aeruginosa and Gram-positive cocci \\
\hline Urinary catheter cystitis & S. epidermidis, K. pneumoniae, E. faecalis, Proteus mirabilis \\
\hline Peritoneal dialysis ( CAPD) peritonitis & A variety of bacteria and fungi \\
\hline IUDs & $\begin{array}{l}\text { S. epidermidis, S. aureus, Corynebacterium sp., Micrococcus sp., } \\
\text { Enterococcus sp., Candida albicans, Group B Streptococci. }\end{array}$ \\
\hline Endotracheal tubes & A variety of bacteria and fungi \\
\hline Hickman catheters & S. epidermidis and C. albicans \\
\hline Central venous catheters & S. epidermidis, S. aureus, E. faecalis, K. pneumoniae, $P$. aeruginosa, C. albicans \\
\hline Mechanical heart valves & Viridans streptococci, Enterococci \\
\hline Vascular grafts & Gram-positive cocci \\
\hline Biliary stent blockage & A variety of enteric bacteria and fungi \\
\hline Orthopedic devices & $\begin{array}{l}\text { Hemolytic streptococci, Enterococci, P. mirabilis, Bacteroides sp., P. } \\
\text { aeruginosa, E. coli }\end{array}$ \\
\hline Pentile prostheses & S. aureus and S. epidermidis \\
\hline
\end{tabular}

The development of these agents did not take into account the unique biology of bacterial groups i.e. formation of biofilms. Antibiotic therapy typically reverses the symptoms caused by planktonic cells released from the biofilm, but fails to kill the biofilms [8]. For this reason biofilm infections typically show recurring symptoms after cycles of antibiotic therapy until the sessile population is surgically removed from the body. Planktonic bacterial cells are released from biofilms and this is a natural pattern of programmed detachment. Thus, biofilms can act as 'niduses' of acute infection. It is likely that biofilms evade anti microbial challenges by multiple mechanisms [1].

1. Failure of an agent to penetrate the full depth of the biofilm.

2. Atleast some of the cells in a biofilm experience nutrient limitation and therefore exist in a slow growing or starved state and these cells are not very susceptible to many antimicrobial agents.

3. Some of the cells in a biofilm adopt a distinct and protected biofilm phenotype which is not a response to nutrient limitation but it is a biologically programmed response to growth on a surface.

\section{Pseudomonas aeruginosa Biofilms}

$P$. aeruginosa is the principal pathogen in the lungs of patients with cystic fibrosis (CF). Chronic colonization by this bacteria leads to progressive lung damage and eventually respiratory failure and death in most CF patients. In $P$. aeruginosa; a complex quorum sensing hierarchy plays a central role in the regulation of virulence and contributes to the late stages of biofilm maturation. P. aeruginosa possess two AHL - dependent quorum sensing systems, termed Las RI and RhlR1. Las 1 and Rhl1 are lux homologs that direct the synthesis of N-(3-oxododecanoyl) homoserine lactone (3-oxo -(12-HSL) and N-butanoylhomoserine lactone (C4-HSL). The target genes regulated via las and rhl overlap considerably and recently a third Lux R homolog (Qsc R) has been identified that further modulates their expression. Apart from AHLS, $P$. aeruginosa also produces a third quorum sensing signal 
molecule which is essential for the expression of many rhl dependent phenotypes as well as biofilms development [9].

Antibiotic therapy in patients colonized with $P$. aeruginosa often gives a measure of relief from symptoms but fails to cure the beset ongoing infection. This is because the antibiotic therapy cannot eliminate the antibiotic resistant sessile biofilm communities.

\section{Staphylococcal Biofilms}

The genetic and molecular basis of biofilm formation in staphylococci is multifaceted. The ability to form a biofilm affords at least two properties: the adherence of cells to a surface and accumulation to form multilayered cell clusters. A trade mark is the production of the slime substance PIA, a polysaccharide composed of beta - 1,6 - linked N-acetyl glucosamines with partly diacetylated residues, in which the cells are embedded and protected against the host's immune defence and antibiotic treatment. Mutations in the corresponding biosynthesis genes (ica operon) lead to a pleiotropic phenotype; the cells are biofilm and haemagglutination negative, less virulent and less adhesive on hydrophilic surfaces. ica expression is modulated by various environmental conditions, appears to be controlled by Sig B and can be turned on and off by insertion sequence (IS) elements.

Proteins have been identified that are also involved in biofilm formation such as the accumulation-associated protein (AAP), the clumping factor A (Clf A), the staphylococcal surface protein (SSP1) and the biofilm associated protein (Bap). Intercellular adhesions with in biofilms of Staphylococcus epidermidis, a major cause of medical device related infections, is mediated by the PIA [10].

\section{Dental Biofilms}

Dental biofilms, more commonly called plaque, are probably the most well studied natural biofilm in humans. Development of dental biofilms follows a sequence of events and involves hundreds of species of bacteria. After a good dental cleaning, tooth enamel becomes coated with a variety of proteins and glycoproteins of host origin. This coating is called as acquired pellicle. Then the primary colonizers, first streptococci and later actinomycetes, colonize the surface of the teeth by adhesion molecules and pilli.

The bacteria on the pellicle undergo cell to cell interaction via quorum sensing. A number of streptococci, including Streptococcus mutans and related organisms, begin to synthesize insoluble glucan via glucan binding protein. Bridge bacteria (members of the genus fusobacterium) form aggregates with primary colonisers. The late colonisers form aggregate with bridge bacteria. At this point of time, the biofilm consists primarily of nonpathogen. However, in the presence of dietary sucrose and other carbohydrate, acids are produced via fermentation, which leads to demineralisation of the tooth enamel, over the time, caries. If the plaque is allowed to remain undisturbed on the teeth for several days, the microbial flora continues to change. The last colonisers of the biofilm are considered pathogenic because of their role in periodontal disease. The most important pathogens include Porphyromonas gingivalis, Bacteriodes forsythus, Actinobacillus actinomycetiemcomitans and Treponema denticola [2,11].

\section{Candida Biofilms}

Most manifestations of candidiasis are associated with the formation of Candida biofilms on surfaces and it is also associated with infections at both mucosal and systemic sites.

Candida biofilms share several properties with bacterial biofilms. C. albicans biofilm formation has 3 distinct developmental phases: early (0-11 h), intermediate (12-30 h) and mature (38-72h) [12]. The detailed structure of mature C. albicans biofilms consists of a dense network of yeast, hyphae and pseudohyphae. This mixture of yeast, hyphae and matrix material is not seen when the organisms is grown in liquid culture or on an agar surface, which suggests that morphogenesis is triggered when an organisms contacts a surface [13-15].

Studies showed that $C$. dubliniensis has the ability to adhere to and form biofilms with structural heterogeneity and typical microcolony and water channel architecture similar to bacterial biofilms and C. albicans biofilms [15,16].

Indwelling intravascular catheters represent a risk factor that is associated with nosocomial Candida infections. The devices become colonised by the microorganisms that form a biofilm of cells, the detachment of which can result in septicaemia [17-19].

Antifungal drug resistance is quickly becoming a major problem. Major genes that contribute to drug resistance in $C$. albicans and C. dubliniensis are CDR genes (CDR 1 and CDR 2 ) and MDR genes. These genes have been demonstrated to be upregulated during biofilm formation and development $[12,13,17]$.

\section{Detection of Biofilms}

With the emergence of biofilm associated diseases, there are considerable diagnostic problems for the clinical laboratory. These problems can be classified into five categories: false negative cultures, visible but non cultivable organisms, underestimated or low colony count, inappropriate specimen and loss of or decreased antimicrobial susceptibility. Biofilms are resilient, adherent and with EPS, quite resistant to culturing by swabs.

\section{Detection in Loose Needle Connectors}

1. Cooper et al. [20] developed a Gram staining technique of the catheter tips, the technique depends on optical properties of the different catheters but it is time consuming since it requires the microscopical examination of atleast 200 oil immersion fields.

2. By direct acridine orange staining of the catheter tips [21].

3. By scanning electron microscope.

4. Maki et al. [22] developed a semiquantitative method for culturing vascular cannulas on solid media. 


\section{Methods}

Tissue Culture Plate Method (TCP)

The TCP assay described by Christensen et al. [23] is most widely used and is considered a standard test for detection of biofilm formation. The microorganisms are grown in polystyrene tissue culture plates for 24 hours then after washing fixed with sodium acetate (2\%) and stained with crystal violet $(0.1 \% \mathrm{w} / \mathrm{v})$. Biofilm formation is detected by measuring optical density with ELISA reader.

\section{Tube Method [24]}

It is a qualitative assessment of biofilm formation where the microorganisms are grown in trypticase soy broth with $1 \%$ glucose in tubes for 24 hours. The tubes are then decanted and washed with PBS (phosphate buffer saline) and stained with crystal violet $(0.1 \%)$. The tubes are then washed and dried and biofilm formation is considered positive when a visible film lines the wall and bottom of the tube.

\section{Congo Red Agar Method (CRA) [25]}

The microorganisms are grown on brain heart infusion agar with 5\% sucrose and congo red. Positive results are indicated by black colonies with a dry crystalline consistency.

\section{Bioluminescent Assay}

\section{Attenuated Total Reflecting Spectroscopy (ATR)}

It has been used to monitor the conditioning films that are an early harbinger of biofilm formation.

\section{Piezoelectric Sensors}

Such as quart with crystal microbalances monitor frequency shifts as mass accumulates on the sensor surface.

\section{Possible Strategies for Eradication of Biofilms [2,26,27]}

For eradication, combination of strategies have been used:

1. Mechanical disruption / removal (sonication);

2. Immune modulation (Azithromycin and low dose doxycycline);

3. Antimicrobial agents (silver and tobramycin);

4. Amphotericin B lipid formulations and the Echinocandins against the Candida biofilms.

The effective control will require a concerted effort to develop therapeutic agents that target the biofilm phenotype and community signalling - based agents that prevent the formation, or promote the detachment of biofilms.

\section{References}

1. Costerton J.W., Stewart P.S., Greenberg E.P. Bacterial biofilms: A common cause of persistent infections.Science 1999;284:1318-22.

2. John G.T., Donale C.L. Biofilms: architects of disease. In: Connie R.M., Donald C.L., George M., editors. Textbook of diagnostic microbiology. $3^{\text {rd }}$ ed. Saunders 2007; p. 884-95.

3. Victoria W., Serralrta B.S. Catherine H., et al. Life styles of bacteria in wounds: presence of biofilms. Wounds 2001;13(1):29-34.
4. Lam J., Chan R., Lam K., Costerton J.W. Production of mucoid microcolonies by Pseudomonas aeruginosa within infected lungs in cystic fibrosis. Infect Immun 1980;28:546-56.

5. Nickel J.C. Bacterial biofilms in urology. Infect Urol 1998;11(6):169-75.

6. Ward K.H., Olson M.E., Lam K., Costerton J.W. Mechanism of persistent infection associated with peritoneal implant. J Med Microbiol 1992;36:406.

7. Cochrane D.M.G. Immune response to bacterial biofilms. Med Microbiol J 1988;27:255.

8. Sritharan M., Sritharan V. Emerging problems in the management of infectious diseases: the Biofilms. Indian J Med Microbiol 2004;22(3):140-2.

9. Hardie K.R., Badwin T., William P. Molecular basis of bacterial adaptation to pathogenic life style. In: Borriello SP, Murray PR, Funke G, editors. Topley and Wilson's Microbiology and Microbial infections. $10^{\text {th }}$ ed. Hodder Arnold, ASM Press, 2005:147-82.

10. Gotz F. Staphylococcus and biofilms. Mol Microbiol 2002;43(6):1367-78.

11. Rosan B. Dental plaque formation. Microbes Infect 2000; 2: 1599.

12. Chandra J., Kuhn D.M., Mukherjee P.K., et al. Biofilm formation by fungal pathogen C. albicans: dev, architec, and drug (R). J Bacteriol 2001;183:5385-94.

13. Douglas L.J. Medical importance of biofilms in Candia infection. Rev Iberam Micol 2002;19:139-43.

14. Douglas L.J. Candida biofilms and their role in infection. Trends Microbiol 2003;11:30-6.

15. Ramage G., Vande Walle K., Wickes B.L., Lopez Ribod J.L. Biofilm formation by Candida dubliniensis. J Clin Microbiol 2001;39:3234-40.

16. O’Toole G.A., Kaplan H.B., Kolter R. Biofilm formation as microbial development. Annu Rev Microbiol 2000;54:49-79.

17. Ramage G., Bachmann S., Patternson T.F., et al. Investigation of multidrug efflux pumps in relation to fluconazole resistance in Candida albicans biofilms. J Antimicrob Chemother 2002;49:973-80.

18. Adam B., Baillie G.S., Douglas L.J. Mixed species biofilms of Candida albicans and Staphylococcus epidermidis. J Med Microbiol 2002;51:344-9.

19. Donlan R.M. Biofilms: microbial life on surfaces. Emerg Infect Dis 2002;8:1-19.

20. Cooper G.L., Hopkins C.C. Rapid diagnosis of intravasculo-catheter associated infection by direct Gram staining of catheter segments. N Engl J Med 1985;312:1142-7.

21. Zufferey J., Rime B., Francioli P., Bille J. Simple method for rapid diagnosis of catheter - associated infection by direct Acridine Orange staining of catheter tips. J Clin Microbiol 1988;26(2):175-7.

22. Maki D.G., Weise C.E., Sarafin H.W. Semiquantitative culture method for identifying intravenous - catheter related infection. N Eng J Med 1977;296:1305-9.

23. Christensen G.D., Simpson W.A., Yonger J.A., et al. Adherence of coagulase negative staphylococci to plastic tissue cultures: a quantitative model for the adherence of staphylococci to medical device. J Clin Microbiol 1985;22:996-1006.

24. Christensen G.D., Simpson W.A., Bisno A.L., Beachey E.H. Adherence of slime - producing strains of Staphylococcus epidermidis to smooth surfaces. Infect Immun 1982;37:318-26.

25. Freeman D.J., Falkiner F.R., Keane C.T. New method for detecting slime production by coagulase negative staphylococci. J Clin Pathol 1989;42:872-4.

26. Bachmann S.P., Vande Walle K., Ramage G., et al. In vitro activity of casofungin against Candida albicans biofilms. Antimicrobiol Agents Chemother 2002;46:3591-6.

27. Kuhn D.M., George T., Chandra J., et al. Antifungal susceptibility of Candia biofilms: unique efficacy of amphotericin B lipid formulation and echinocandins. Antimicrobial Agents Chemother 2002;46:1773-80. 